\title{
LETTERS
}

\section{Clarifying the role of lung ultrasonography in COVID-19 respiratory disease}

In their CMAJ article, Thomas and colleagues ${ }^{1}$ describe a patient with pneumonia associated with coronavirus disease 2019 (COVID-19) in whom lung ultrasonography showed "multifocal B-lines, pleural thickening and subpleural consolidation." Based on these findings, the authors proposed "that lung ultrasonography may be useful in the workup of patients with suspected COVID-19, even though differentiating between different causes of viral pneumonia is not possible." These comments require further clarification.

First, the sonographic findings described here are highly nonspecific: they are found not only in other viral pneumonias but also in nonviral pneumonias and a wide spectrum of noninfectious processes, including interstitial lung disease and acute respiratory distress syndrome (ARDS). ${ }^{2}$

Second and consequently, the nature of their patient's sonographic findings must be further qualified, in particular the presence of multifocal B-lines. If by this term 3 or more B-lines per acoustic window is designated, an "interstitial" (or "alveolar-interstitial") pattern is identified (whereas occasional B-lines, especially dependently, can be normal). ${ }^{3}$ However, if by this term a certain global distribution of interstitial pattern is designated, diagnostic implications follow: whereas a homogeneous interstitial pattern favours cardiogenic edema, a heterogeneous interstitial pattern, particularly in combination with subpleural consolidation, pleural thickening and reduced lung sliding, is consistent with pneumonia or ARDS. ${ }^{4}$ Presuming a heterogeneous interstitial pattern, this patient's overall presentation suggests concomitant COVID-19 pneumonia and ARDS.

The findings on lung ultrasonography of COVID-19 respiratory disease appear to be nonspecific and likely are on par with similar non-COVID respiratory diseases; the need for microbiological confirmation remains. In general, lung ultrasonography in critical illness provides information that is probably best regarded as complementary to radiography of the chest. The unique benefit of lung ultrasonography in the current context includes bedside feasibility, in particular when advanced chest imaging is unavailable or contraindicated for infection control. ${ }^{5}$

\section{Cameron W. Pierce MA MD}

Respirologist, Vancouver Coastal Health, Vancouver, BC

Cite as: CMAJ 2020 April 20;192:E436. doi: $10.1503 / \mathrm{cmaj} .75311$

\section{References}

1. Thomas A, Haljan G, Mitra A. Lung ultrasound findings in a 64-year-old woman with COVID-19. CMAJ 2020;192:E399.

2. Mojoli F, Bouhemad B, Mongodi S, et al. Lung ultrasound for critically ill patients. Am J Respir Crit Care Med 2019;199:701-14.

3. Doerschug KC, Schmidt GA. Intensive care ultrasound: III. Lung and pleural ultrasound for the intensivist. Ann Am Thorac Soc 2013;10:708-12.

4. Copetti R, Soldati G, Copetti P. Chest sonography: a useful tool to differentiate acute cardiogenic pulmonary edema from acute respiratory distress syndrome. Cardiovasc Ultrasound 2008;6:16.

5. See KC, Ong V, Tan YL, et al. Chest radiography versus lung ultrasound for identification of acute respiratory distress syndrome: a retrospective observational study. Crit Care 2018;22:203.

Competing interests: None declared. 